\title{
ACRF Instrumentation Status and Information
}

JW Voyles

August 2009

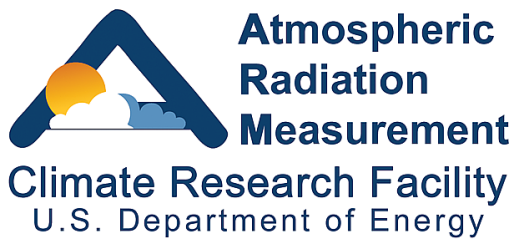

Work supported by the U.S. Department of Energy, Office of Science, Office of Biological and Environmental Research 


\section{DISCLAIMER}

This report was prepared as an account of work sponsored by the U.S. Government. Neither the United States nor any agency thereof, nor any of their employees, makes any warranty, express or implied, or assumes any legal liability or responsibility for the accuracy, completeness, or usefulness of any information, apparatus, product, or process disclosed, or represents that its use would not infringe privately owned rights. Reference herein to any specific commercial product, process, or service by trade name, trademark, manufacturer, or otherwise, does not necessarily constitute or imply its endorsement, recommendation, or favoring by the U.S. Government or any agency thereof. The views and opinions of authors expressed herein do not necessarily state or reflect those of the U.S. Government or any agency thereof. 


\begin{abstract}
The purpose of this report is to provide a concise but comprehensive overview of Atmospheric Radiation Measurement Climate Research Facility instrumentation status. The report is divided into the following five sections: (1) new instrumentation in the process of being acquired and deployed, (2) field campaigns, (3) existing instrumentation and progress on improvements or upgrades, (4) proposed future instrumentation, and (5) Small Business Innovation Research instrument development. New information is highlighted in blue text.
\end{abstract}




\section{Acknowledgments}

This report is developed largely from the information submitted to and managed within our Instrument Mentor Monthly Summary (IMMS) reporting system (http://www.db.arm.gov/IMMS/). Special thanks to our Instrument Team for providing timely and complete updates to the IMMS, to Kathy Doty, our developer and administrator of IMMS, and Rolanda Jundt, who ensures this information is posted accurately on the ARM website. 


\section{Contents}

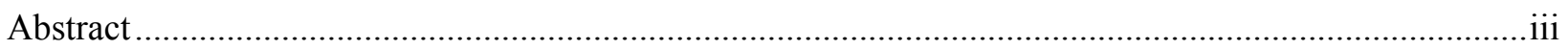

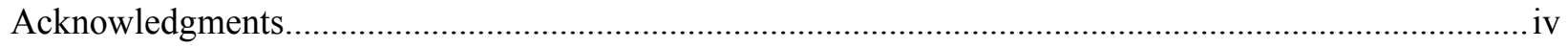

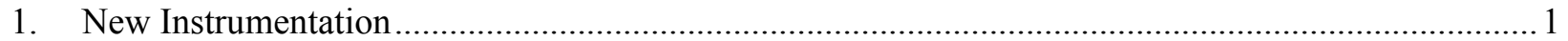

1.1 ACRF Initiative - Recovery Act Instrumentation ............................................................... 1

1.2 Rotating Shadowband Spectrometer Overhaul .............................................................. 1

1.3 Add Scanning Capability to the W-Band ARM Cloud Radar ............................................ 1

1.4 Next-Generation Microwave Radiometers ..................................................................... 1

$1.5 \quad$ Photoacoustic Soot Spectrometer ……........................................................................ 1

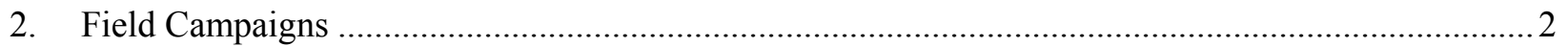

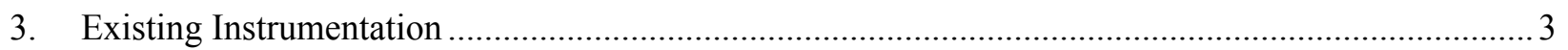

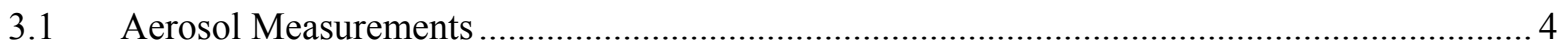

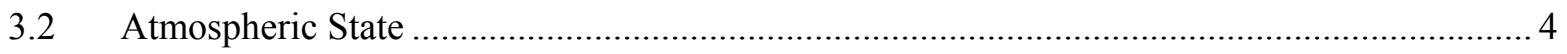

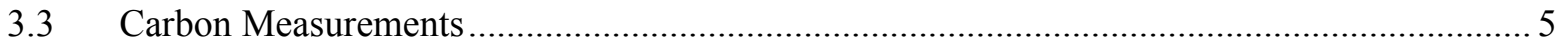

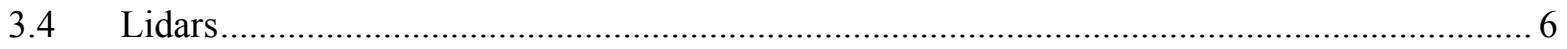

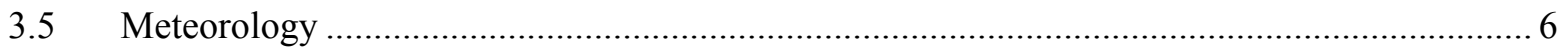

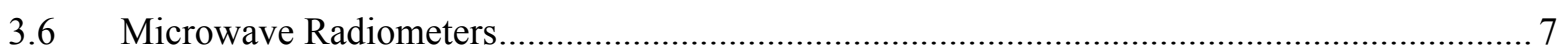

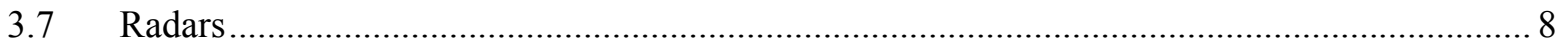

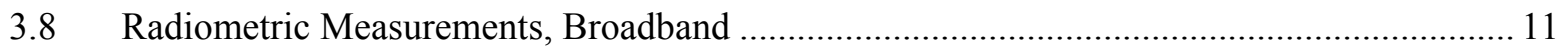

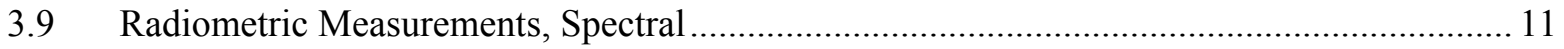

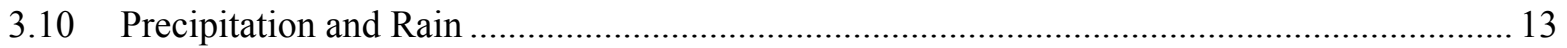

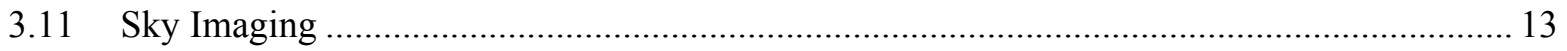

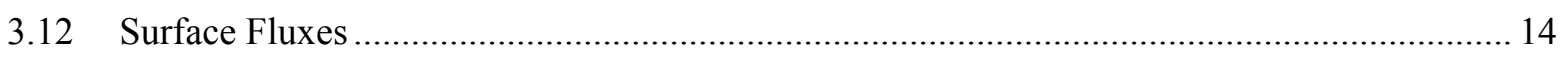

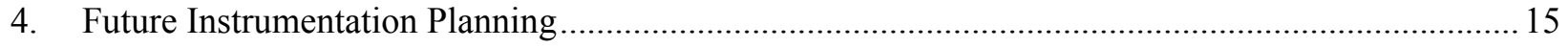

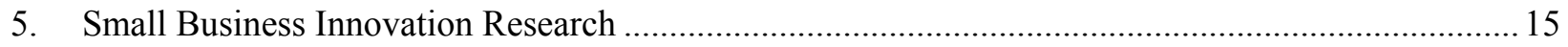




\section{New Instrumentation}

This section describes approved new instrument deployment and upgrade activities for the current fiscal year (FY).

\subsection{ACRF Initiative - Recovery Act Instrumentation}

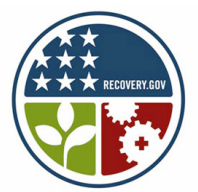

Through the American Recovery and Reinvestment Act of 2009, the Department of Energy's Office of Science received $\$ 1.2$ billion. In late May, DOE released approximately $\$ 54$ million — 90 percent — of the $\$ 60$ million allocated to the ARM

Climate Research Facility. The remaining 10\% of the Recovery Act funds ( $\$ 6$ million) was released in August. During the next 18 months, the ACRF will purchase and deploy dual-frequency scanning cloud radars to all the ACRF sites, enhance several sites with precipitation radars and energy flux measurement capabilities, and invest in new aerosol sampling and aerial instrumentation. This is just a small sample of the planned enhancements that will result in 143 new instruments for the ACRF user community.

Contact Jimmy Voyles for specific details related to Recovery Act tasks.

\subsection{Rotating Shadowband Spectrometer (RSS) Overhaul}

Please refer to Engineering Change Order ECO-00661, RSS Refurbishment, in the RSS section of this report.

\subsection{Add Scanning Capability to the W-Band ARM Cloud Radar (WACR)}

Please refer to Engineering Change Order ECO-00658, WACR-Add Scanning Capability, in the W-Band ARM Cloud Radar (WACR) section of this report.

\section{$1.4 \quad$ Next-Generation Microwave Radiometers (MWRs)}

Please refer to Engineering Change Order ECO-00664, Next Generation MWR Procurement/Deployment, in the Microwave Radiometers (MWR) section of this report.

\subsection{Photoacoustic Soot Spectrometer (PASS)}

The Science and Infrastructure Steering Committee (SISC) approved the Working Group's recommendations to deploy an instrument that provides photoacoustic extinction of aerosols. The PASS instrument and associated measurements will be added to the existing ACRF Aerosol Observing System (AOS) at the Southern Great Plains (SGP). A science objective is to produce a high-quality data set to investigate the reported bias in the absorption measurements made by the particle/soot absorption photometer instruments.

Engineering Change Order ECO-00663, Photoacoustic Instrument to Enhance Aerosol Optical Data Quality at ARM, is approved and in process to document this specification, procurement, and deployment. Dubey Manvendra is the leader. The procurement requisition is complete and discussions are ongoing 
related to field installation details. The PASS instrument has been received and is undergoing characterization. The PASS system is installed and operational. Data collections are operational and ingest development is ongoing.

\section{Field Campaigns}

Contact: Jimmy Voyles, ACRF Instrument and Field Campaign Coordinator

This section provides information for ACRF field campaigns that have a significant impact on instrumentation and instrument team resources. Please refer to the ACRF field campaign web page at http://www.arm.gov/acrf/fc.stm for approved activities.

FY2008-2009

ACRF- Chile; RHUBC-II 2009. Engineering Work Order EWO-12438, Radiative Heating in Underexplored Bands Campaign 2 (RHUBC-II), documents instrumentation requirements for this experiment. The Radiative Heating in Underexplored Bands Campaign (RHUBC-II) was selected for support by ACRF as an offsite campaign. Led by principal investigators David Turner and Eli Mlawer, RHUBC-II will take place from August to October 2009 at a location near Cerro Chajnantor in Chile, at an altitude of more than $5000 \mathrm{~m}$. This effort is a follow on to RHUBC-I, conducted from February 22 to March 14, 2007, at the ACRF North Slope of Alaska (NSA) site in Barrow. During RHUBC-II, the same spectral band will be explored; however, this absorption band is much more transparent in the dry, lowpressure conditions at Chajnantor. These conditions represent the upper troposphere that is poorly observed, but important for climate studies. Therefore, significant fundamental advances that are pertinent to reducing uncertainties in the radiation calculation of global climate models will be gained.

FY2010-2011

ACRF AMF1- Azores; Clouds, Aerosol and Precipitation in the Marine Boundary Layer (CAP-MBL). Scientists will take advantage of the user facility's ARM Mobile Facility - a heavily instrumented portable atmospheric laboratory - to study low marine clouds and aerosols in the Azores. Led by Dr. Robert Wood from the University of Washington, the new award extends the original 9-month project duration on Graciosa Island from March 2009 to November 2010. Doubling the length of the deployment will provide significantly greater statistical reliability of the relationships between aerosol and cloud properties required for evaluation of climate and process models.

ACRF- Alaska; Arctic Lower-Troposphere Observed Structure (ALTOS). This 2-month campaign in 2010 will focus on the fall transition season, when sea ice begins to form and dramatic changes in aerosol and cloud properties occur. Led by Dr. Johannes Verlinde from the Pennsylvania State University, the science team will use a heavily instrumented tethered balloon to make routine ascents and descents in the lower 2 kilometers of the atmosphere at Oliktok Point, Alaska. Supplemented with measurements from an instrumented ground station, this campaign will be the first to capture a full atmospheric profile of in situ cloud microphysics, aerosols, and radiative measurements during the Arctic transition season. The unique data set will provide a thorough case for testing Arctic cloud processes used in climate models and for testing the algorithms used to retrieve these measurements. 
ACRF AAF- SGP; Small Particles in Cirrus Clouds (SPARTICUS). Mid-latitude cirrus clouds are the focus of this study that will use instrumented aircraft to sample clouds above the user facility's SGP site in Oklahoma. Led by Dr. Jay Mace from the University of Utah, this field campaign will occur between October 2009 and June 2010, spanning the time of year when cirrus clouds are shown to be most prevalent above the site. Data from the campaign will help scientists to address many outstanding questions regarding mid-latitude cirrus properties and processes and to validate and implement improved algorithms for measurements of cirrus clouds at all the user facility's research sites.

An intensive phase of the project will focus more on microphysical processes and field a more extensive set of experimental probes that observe the aerosol and ice nuclei properties of the upper troposphere. DOE and NASA will jointly conduct the intensive phase of the experiment.

\section{ACRF AMF2- Colorado; Storm Peak Lab Cloud Property Validation Experiment (STORMVEX).}

Dr. Mace is also the lead scientist for the study of liquid and mixed-phase clouds at the Storm Peak Laboratory near Steamboat Springs, Colorado. This campaign will debut the user facility's second mobile unit, which is currently under development. It will be deployed from approximately October 2010 through March 2011 at a location near the laboratory, which operates at an elevation of 3210 meters above sea level. The close proximity of the mobile facility and the laboratory's instrument platform is expected to result in a correlative data set equivalent to between 200 and 300 aircraft flights in liquid and mixed-phase clouds.

\section{Existing Instrumentation}

This section describes activities that are ongoing to improve the performance or maintain existing instrumentation, including any planned or in-progress upgrades. The information is abstracted primarily from the Instrument Mentor Monthly Summary (IMMS) reports database (http://www.db.arm.gov/IMMS/), which can be used for a collective and historical view of instrument status. Individual IMMS reports may be reviewed by following links to specific instruments from instrument web pages (http://www.arm.gov/instruments). ACRF Mentors provide updates to the IMMS under these categories:

1. Data Review

2. Instrument Performance Issues and Trends

3. Current Task Status

4. Near-term Plans

5. Accomplishments.

Information related to the progress and status of instrument engineering is available from our Engineering Change Order (ECO) database (http://eco.arm.gov).

Helpful links to instrument-related ACRF web pages are listed below:

- Instrument Systems and Mentors, http://www.arm.gov/instruments/mentors.php

- Instrument Locations, http://www.arm.gov/instruments/location.php

- Instrument Mentor Monthly Summaries, http://www.db.arm.gov/IMMS/ 
- Data Quality Reports, http://www.db.arm.gov/cgi-bin/PIFCARDQR2/SignIn.pl?form=dqr

- Data Quality Program, http://dq.arm.gov/.

\subsection{Aerosol Measurements}

\section{Aerosol Observing System (AOS)}

Mentor: John Ogren and Anne Jefferson, NOAA/ESRL/Global Monitoring Division (GMD)

There are no open engineering tasks related to the AOS instrument suite.

\section{Tandem Differential Mobility Analyzer (TDMA)}

Mentor: Don Collins, Texas A\&M University

Engineering Change Request ECO-00640, Replace AOS Optical Particle Counter with Aerodynamic Particle Sizing Spectrometer, is in process. The ARM Working Groups and ARM Science Team Executive Committee (now known as the SISC) approved the addition of an aerodynamic particle-sizing spectrometer (APS) in FY2008 to replace the Optical Particle Counter (OPC) component of the TDMA. Don Collins, TDMA Instrument Mentor, has responsibility for integrating the APS with the SGP AOS. The APS has now been installed and is functioning properly (thanks largely to Pat Dowell). A permanent shelf is being constructed and will be shipped to SGP soon. The TDMA control software has been updated to record and graph the APS data. A few initial glitches had to be worked through, but everything seems to be working very well now. The unprocessed size distributions measured by the TDMA and APS agree surprisingly well in the overlap size range. Work has begun on the software that will be used to process the two data sets together and merge them into a single size distribution.

Engineering Change Request ECO-00587, Develop Collection and Ingest for TDMA. Data from the TDMA currently are acquired and processed by Don Collins. Processed data are then delivered to ACRF on a monthly basis and stored in the IOP area of the ACRF Data Archive as "beta data." An ingest is being developed to produce netCDF files for inclusion in the main Data Archive. The communications group is contacting Don Collins to develop a web area, enter instrument metadata, and edit the instrument handbook. The TDMA needs to have an entry added to the IMMS reporting system. TDMA raw data are now available at the Data Archive.

\subsection{Atmospheric State}

\section{Balloon-Borne Sound System (BBSS)}

Mentor: Rich Coulter (with Mike Ritsche and Donna Holdridge), Argonne National Laboratory

Engineering Change Request ECO-00769, Add Cryogenic Frostpoint Hygrometer Launches at the SGP in support of GRUAN, is approved. Mike Ritsche is the lead. The GCOS Reference Upper Air Network (GRUAN) is an international reference-observing network, designed to meet climate requirements and to fill a major void in the current global observing system. Upper air observations within the GRUAN network will provide long-term high-quality climate records, will be used to constrain and validate data from space-based remote sensors, and will provide accurate data for the study of atmospheric processes. GRUAN has been identified by the climate community as being required to generate long-term high 
quality climate records, to constrain and calibrate data from more spatially comprehensive global observing systems (including satellites and current radiosonde networks), and to fully characterize the properties of the atmospheric column. The GRUAN requirement is to be able to provide one cryogenic frostbite hygrometer (CFH) sounding per month. The ACRF is working with NOAA and the GRUAN Lead Center to establish support for the launches at the SGP by providing base stations, supplies, balloons, gases, cryogenics, and storage containers for cryogenics, calibration supplies, and CFH sondes to obtain water vapor profile measurements into the lower stratosphere once per month.

Engineering Change Order ECO-00691, Update all Digi-Cora-1 and DigiCora-II Chipsets, is approved for FY2008 implementation and is in process. Donna Holdridge is the leader. Due to changes with Vaisala radiosonde booms, new radiation correction tables must be added via updated chipsets. The radiation corrections are required due to the increase in structural integrity of the temperature $(\mathrm{T})$ and relative humidity $(\mathrm{RH})$ booms. Increased surface area leads to an increased solar heating on the booms. The new chipset corrects for this increased heating. DigiCora-III systems already have the new software upgrades that address this issue. This request is only to upgrade spares and backup systems, which are used when the DigiCora-IIIs fail, or for field campaigns, such as the upcoming RHUBC-II. To date, four of the eight systems have been upgraded. The NSA DigiCORA-II was upgraded by Mike Ritsche while he was onsite in June. One of the spare DigiCORA-II systems was upgraded while it was at Vaisala for repair. Two DigiCORA-I systems have been upgraded so far. Tim Grove is in the process of upgrading the remainder of the systems resident at the SGP site.

Engineering Change Request ECR-00694, Characterize the Temperature Dependency of the RH Sensor in the SurTHRef System, is under review. Mike Ritsche is the leader. The goal, if initial analysis and review show promise, are to improve the initial conditions ( $T, R H)$ of the RS-92 sondes at launch timewhile providing NIST tractability of sensor calibrations. This request is on hold until further analysis and results of the technique are available.

\section{SuomiNet Global Positioning System (SuomiNet)}

Mentor: None (external data provided by SuomiNet/COSMIC). Rick Wagener, Brookhaven National Laboratory, is the infrastructure contact.

Please see http://www.unidata.ucar.edu/data/suominet/ and http://www.arm.gov/xds/static/suomigps.stm for the details on the SUOMIGPS data.

\subsection{Carbon Measurements}

\section{Carbon Dioxide Flux System $\left(\mathrm{CO}_{2} \mathrm{FLX}\right)$}

Mentor: Marc Fischer, Lawrence Berkeley National Laboratory

There are no open engineering tasks related to the Carbon Dioxide Flux System (CO2FLX) instrument suite.

\section{Carbon Monoxide (CO) System}

Mentor: Sébastien Biraud, Lawrence Berkeley National Laboratory

There are no open engineering tasks related to the Carbon Monoxide (CO) instrument suite. 


\section{Precision Carbon Dioxide Mixing Ration System (PGS)}

Mentor: Sebastien Biraud, Berkley National Laboratory

There are no open engineering tasks related to the Precision Carbon Dioxide Mixing Ration System (PGS) instrument suite.

\subsection{Lidars}

\section{Micropulse Lidar (MPL)}

Mentor: Rich Coulter, Argonne National Laboratory

Engineering Change Request ECR-00698, Update MPLs to Fast Switching Polarization, submitted by Rich Coulter is approved. The ECR proposes to upgrade each of the ACRF's MPLs to add fast switching polarization. If approved, a plan will be developed to have Sigma Space modify all six installed MPL systems.

\section{Raman Lidar (RL)}

Mentor: Rob Newsom, Pacific Northwest National Laboratory

There are no open engineering tasks related to the Raman Lidar (RL) instrument suite.

\section{Vaisala Ceilometer (VCEIL)}

Mentor: Vic Morris, Pacific Northwest National Laboratory

There are no open engineering tasks related to the Vaisala Ceilometer (VCEIL) instrument suite.

\subsection{Meteorology}

\section{Surface Meteorological Instrumentation}

Mentor: Mike Ritsche, Argonne National Laboratory (SMET, SMOS, SURTHREF, THWAPS, MET, METTWR [NSA Site])

Engineering Change Request ECR-00672, Upgrade Dynamic Rain Gauge Calibration System, is approved. This task is in process to improve the characterization and performance of ACRF's precipitation measurements. Component evaluation and testing continues.

\section{Tower - Meteorological Tower Systems (TWR)}

Mentor: David Cook, Argonne National Laboratory

There are no open engineering tasks related to the TWR instrument suite. 


\subsection{Microwave Radiometers}

\section{Microwave Radiometer (MWR)}

"The MWR Provides microwave radiances (expressed as "brightness temperatures") obtained in the range of 23.8 and $31.4 \mathrm{GHz} . "$

Mentor: Maria Cadeddu, Argonne National Laboratory

There are no open engineering tasks related to the MWR instrument suite.

\section{Microwave Radiometer 3 Channel System (MWR3C) (Next Generation MWR)}

"The MWR Provides microwave radiances (expressed as "brightness temperatures") obtained in the range of 22 to 30 and $90 \mathrm{GHz} . "$

Mentor: Maria Cadeddu, Argonne National Laboratory

Engineering Change Order ECO-00664, Next-Generation MWR Procurement/Deployment, is approved for action. The ARM Working Groups and ARM Science Team Executive Committee (now known as the SISC) have approved the competitive procurement of next-generation 3-channel microwave radiometers (MWR3Cs). The systems are specified to provide three channels operating at 23, 31, and $90 \mathrm{GHz}$. The strategy is to replace the current aging MWRs with systems that broaden ACRF's measurement performance parameters and provide an economic product life cycle for the future. A procurement specification is in development based on the outcomes of the November 2007 "ACRF MWR Futures" workshop. The contract for the MWR3C was awarded to Radiometrics Corporation. A preliminary design review was conducted at the Radiometrics facility in Boulder, CO. Component availability for the $89 \mathrm{GHz}$ receiver is causing some delay in the build and integration of the instrument. The pan-tilt positioner has been received and is being integrated in the design. The software is almost complete; the RF deck, lenses, and antenna systems are completed. The enclosure required for the IRT is ready for integration and rain mitigation subsystem design will continue as final dimensions are finalized. The first system is scheduled for delivery in September 2009. The second system will follow shortly.

\section{High-Frequency Microwave Radiometer (MWRHF)}

"Provides microwave radiances (expressed as "brightness temperatures") obtained in the range of 90-150 Ghz.

Mentor: Maria Cadeddu, Argonne National Laboratory

There are no open engineering tasks related to the MWRHF instrument suite.

NOTE: The two high-frequency microwave radiometers (MWRHFs) are new instruments from the Radiometer Physics GmbH that are still under testing. 


\section{Microwave Radiometer Profiler (MWRP)}

"Provides microwave radiances (expressed as "brightness temperatures") obtained at twelve frequencies in the range of 22-30 GHz (K-band) and 51-59 GHz (V-band)."

Mentor: Maria Cadeddu, Argonne National Laboratory

There are no open engineering tasks related to the MWRP instrument suite from Radiometrics.

\section{Microwave Radiometer, G-Band (183.3 GHz) Water Vapor Radiometer (GVR)}

"Provides microwave radiances (expressed as "brightness temperatures") obtained in the range of $183.3 \mathrm{GHz}$.

Mentor: Maria Cadeddu, Argonne National Laboratory

There are no open engineering tasks related to the G-band (183 GHz) Vapor Radiometer (GVR) instrument suite from ProSensing Inc.

There is also a G-band (183 GHz) Vapor Radiometer Profiler (GVRP) radiometer developed by Radiometrics under the U.S. Department of Energy (DOE) Small Business Innovative Research (SBIR) program. This system is also known as the MP183. The GVRP has 15 channels between $170 \mathrm{GHz}$ and $183.31 \mathrm{GHz}$. This system has not completed the ACRF baseline processes.

\subsection{Radars}

\section{Millimeter Wave Cloud Radar (35 GHz) (MMCR)}

Mentor: Kevin Widener, Pacific Northwest National Laboratory; Karen Johnson, Brookhaven National Laboratory

Engineering Change Order ECO-00680, MMCR - External Calibration Source, is approved and assigned to Kevin Widener to design a relative calibration source that can be mounted outside the field of view of MMCR antennas. If successful, this enhancement will provide an indication that the sensitivity of the radar receivers is changing. This ECO is on hold pending execution of Recovery Act efforts to update the MMCR processors.

Engineering Change Order ECO-00655, MMCR - End-to-End Calibration Analysis, is in process to provide a calibration study of the ACRF MMCR systems, as recommended and approved by the ARM Science Team Executive Committee (now known as the SISC) for FY2008. A contract was awarded to ProSensing to perform an end-to-end characterization of the MMCR transmitter/receiver calibration. There are two analysis tasks related to this ECO; one for the MMCR and one for the WACR. The WACR analysis is complete. Kevin Widener has recently received the draft calibration analysis for the MMCR, which is under review.

Engineering Change Order ECO-00552, Barrow MMCR-Polarization Upgrade, is on hold (waiting). Because the PIRAQ-III processor does not support polarization, the installation of the orthomode transducer at Barrow is on hold while calibration issues are being reconciled. 
Engineering Change Order ECO-00551, Refurbish Millimeter Wave Cloud Radar Antennas, was begun in 2007, and over a 3-year period, the MMCR antennas will be refurbished and characterized on an antenna range. The spare antenna is complete and the contract for the new feed and subreflector has been placed. Once these are completed, they will be installed on the antenna reflector and calibrated. The Barrow MMCR antenna will be refurbished first to avoid impacting planned field campaigns at SGP. Contract negations are in process for new antennas with a new contractor. For FY2008, two antennas with engineering and fabrication costs are approved for procurement and installation at the SGP and TWP sites. The SGP antenna is 10 foot in diameter, while antennas used at ACRF's tropical sites are 6 foot in diameter. Two additional antennas are planned for FY2009 to support upgrades to ACRF's TWP sites. The first two antennas ( 6 feet) are about to be delivered from Millitech, Inc. The first will replace the 10-feet antenna while it is being evaluated for refurbishment at Millitech. If it is "refurbishable", it will get a new feed, subreflector, and radome and won't be gone too long from the site. If not, Millitech will build a new antenna, which will take considerably longer ( $\sim 6-7$ months). Either way, once a good 10-feet antenna is back, we will reinstall it and forward the 6-foot antenna currently being used at SGP to Darwin. Antennas were damaged in route to the SGP and are being returned to the vendor, Millitech, for re-characterization. Turnaround time is estimated to be 3 weeks for the 6 -foot antennas. When the 10 -foot antenna is sent for refurbishment or new manufacture, the turnaround time will be approximately 4-5 months for refurbishment or approximately 6-8 months if we have to have a new antenna built.

Engineering Change Order ECO-00420, Software I/Q Balancing on MMCRs, was approved to remedy a problem with balance of the MMCR I and Q channels. This slight imbalance makes spectral analysis very difficult under certain conditions. Radar data provided by the site scientist office at NSA are being analyzed to evaluate software fixes. Millitech has determined that their antenna test range will not be able to handle the 10' antenna for characterization. They found two other test ranges (Air Force) that are available for rent. However this increases the cost of characterization from $\sim \$ 7 \mathrm{~K}$ to $\sim \$ 40 \mathrm{~K}$.

Kevin surveyed a select group from the ARM Radar Group and the consensus is that we should keep a $10^{\prime}$ antenna at SGP and buy a new one without characterizing the old antenna. We place a new contract with Millitech for a new 10' antenna and a 6' antenna. The two 6' antennas that we have now will be sent to Manus and Darwin for installation in September/October 2009.

Engineering Change Order ECO-00391, Millimeter Wave Cloud Radar Spectra Processing, is in process to filter the spectra files produced by the upgraded MMCRs (C40 or PIRAQ-III processors), which range from 8 to 15 GB per day. Engineering Change Order ECO-00575, Study Network Transfer of MMCR and WACR Spectra to Archive, is active for data system engineering to support this task. Algorithms for eliminating clear-sky periods and compressing the files need to be developed and implemented locally. The data are collected, processed, and shipped hourly. The MMCR spectra compression software has been running at the SGP site since October 1, 2007. Baseline Change Request BCR-01301, Install MMCR_Spec_Filter (Compress Spectra for MMCR), tracks this effort. The compression results are monitored via plots posted at http://c1.dmf.arm.gov/data/process/sgp/sgpmmcrspecmaskC1.a0/2007/. SGP is complete. This ECO will remain open until NSA and TWP are updated with the new processing.

Overall, the results look very good. There is concern that spectra for some very thin potential clouds are being removed. Approaches to identify these features and retain the spectra at such time-height points without saving very large hydrometeor-free regions of data are under evaluation. All raw (uncompressed) 
spectra data are being retained for 90 days to allow time to review the compression results. Discussion is ongoing to determine when the shipping of spectra on hard drives will be halted.

\section{W-Band (95-GHz) ARM Cloud Radar (WACR)}

Mentor: Kevin Widener, Pacific Northwest National Laboratory

Engineering Change Order ECO-00681, SWACR: Sampling Strategy, Software, Products, is approved and assigned to Pavlos Kollias to define scanning strategies, the operational modes, and the value-added products (VAP) envisioned to produce out of the SWACR. The final statement of work with Pavlos is in place.

Engineering Change Order ECO-00658, WACR-Add Scanning Capability, is in process to plan the initial science, operational, and technical objectives of the development and lead to a procurement specification. Kevin Widener is the leader. During the December 2007 ARM Science Team Executive Committee (now known as the SISC) meeting, a scientific and programmatic consensus was reached to add a scanning capability to the SGP WACR. This enhancement to the WACR will provide an ACRF product for evaluating the 3D cloud properties first at the SGP site and then, depending on portability (or transportability) and robustness, other ACRF research sites. The contract with ProSensing is placed and the scanner is being procured. Removal and shipment of the WACR from the SGP site to ProSensing is complete. The target return date to the SGP is March/April 2009 for evaluation prior to shipping the system with the AMF2 (or other field campaign support) experiment in FY2010. A preliminary design review was conducted and documented in the ECO. A design review was conducted in November. The system design is acceptable and on track. A narrow field-of-view camera and IRT (narrowband centered around 11 microns) are being added in a co-pointing configuration with the radar. The data system engineering team is preparing the ingest. The antenna positioner has arrived at ProSensing and the completion date has been extended to June/July due to component delay. Design for the SWACR installation pad (next to the SGP MMCR shelter) was completed and is being procured. Wiring is progressing and the radar has been assembled. The pad at SGP is complete. We are now planning on going directly from acceptance tests at ProSensing to Graciosa Island, Azores.

Engineering Change Order ECO-00654, WACR-Spectra Notch Filter. The WACR has a software deficiency in the way that the direct current (DC) signal is processed in the Fast Fourier Transform (FFT). ProSensing has identified a method for handling this by incorporating a notch filter taking many averages of the I/Q signal and using this in the FFT. The datastream will remain unchanged. The software was installed and tested on the SGP WACR and will be installed on the AMF WACR as the system is routed through ProSensing from China on the way to the Azores.

Engineering Change Order ECO-00391, Millimeter Wave Cloud Radar Spectra Processing, proposes an evaluation of the feasibility of implementing data reduction algorithms at each MMCR and WACR installation and shipping the resulting files to the ACRF Data Archive via the Internet. A version of this software is in the release process to test ECO-00391 and ECO-00575. Implementation is underway and documented in BCR-1349, Install WACR_Spec_Filter in Production.

\section{Radar Wind Profiler - 915, 1290 MHz (RWP)}

Mentor: Rich Coulter, Argonne National Laboratory 
There are no open engineering tasks related to the 915- and 1290-MHz Radar Wind Profilers (RWPs) instrument suite.

\subsection{Radiometric Measurements, Broadband}

\section{Broadband Radiometer Station (BRS, SIRS, SKYRAD, GNDRAD, BSRN)}

Mentor: Tom Stoffel, National Renewable Energy Laboratory

There are no open engineering tasks related to the Broadband Radiometry instrument suite.

\section{Narrow Field-of-View (NFOV) Radiometer}

Mentor: Gary Hodges, NOAA/ESRL/GMD

There are no open engineering tasks related to the Narrow Field-of-View (NFOV) instrument suite.

\section{Infrared Thermometer (IRT)}

Mentor: Vic Morris, Pacific Northwest National Laboratory

Engineering Change Order ECO-00616, Install IRTs in Ventilated Enclosures, is in process to update our IRT enclosures. In implementing ECO-00345, Install Zenith-Pointing IRT Network at SGP, a HEPAfiltered, ventilated enclosure for the IRTs was designed that keeps debris and, incidentally, most rain, off the gold mirror and IRT lens. This enhancement is being implemented on the Tropical Western Pacific (TWP) and NSA IRT instruments. Vic Morris is leading this ECO. Danny Nelson, Jeff Zirzow, and Krzystof Krzton are tasked under Morris's direction to provide designs for SGP, NSA, and TWP respectively. Morris has recommended an enclosure solution for TWP, please reference details within the ECO.

This ECO has been completed. The final upgrades to the NSA and TWP enclosures are tracked under EWO-12790, Design IRT Enclosure for NSA, and EWO-12791, Design IRT Enclosure for TWP, respectively.

Engineering Change Order ECO-00368, Increase Sample Rate of Infrared Thermometers, is in process to increase the IRT sampling rate to $5 \mathrm{~Hz}$. All systems are functioning except Nauru (see EWO-12288, Update IRT Data Acquisition Software). Communications latency issues are being resolved with the RocketPorts and fiber optic line drivers that are resulting in data collection problems.

\subsection{Radiometric Measurements, Spectral}

\section{Atmospherically Emitted Radiance Interferometer (AERI)}

Mentor: Dave Turner, Space Science and Engineering Center, University of Wisconsin

There are no open engineering tasks related to the Atmospherically Emitted Radiance Interferometer (AERI) instrument suite. 


\section{Cimel Sun Photometer (CSPOT)}

Mentor: None (external data provided by NASA AERONET). Infrastructure contact is Laurie Gregory at Brookhaven National Laboratory.

There are no open engineering tasks related to the Cimel Sun Photometer (CSPOT) instrument suite.

\section{Multi-Filter Rotating Shadowband Radiometer and Related Systems (MFRSR, MFR)}

\section{Mentor: Gary Hodges, NOAA/ESRL/GM Division}

Engineering Change Request ECO-00350, MFRSR Integrating of Campbell Data Logger, is in process and assigned to Gary Hodges to modernize the data acquisition systems for the multi-filter rotating shadowband radiometer (MFRSR) instruments. All SGP instruments are updated - a few lingering communication issues are being resolved. The TWP and AMF site upgrades are planned next and will complete this ECO. The NSA upgrades will be handled by a new ECO and are under evaluation in ECR-00688 due to the software revisions required to allow operation at high latitudes. A separate ECR (00692) is in discussion to complete the build and/or procurement of MFRSR and MFR spare components.

Engineering Change Order ECO-00659, Add Two MFRs to the AMF Instrument Suite, was entered to guide and document the addition of upwelling MFRs to the AMF. Gary Hodges is the leader. Some ramp up to provide this measurement is expected this year. The first radiometer will be installed and verified during the AMF Azores experiment in FY2009, even though surface albedo at the AMF main site will not be representative of the local scale due to the dominant effects of the surrounding sea surface. A second upwelling MFR is proposed for addition to the AMF supplemental site during FY2010; a spare MFR head will be purchased then.

Engineering Change Request ECR-00688, Add Functionality for MFRSR Campbell System at Latitudes $>50 \mathrm{deg}$, submitted by Gary Hodges is approved for a FY2010 implementation. This revision to the MFRSR logger software will provide the capability of the MFRSRs to operate at latitudes greater than 50 degrees latitude. Also, there are ongoing discussions related to an enhancement of the shadowband positioning to enable higher quality retrievals of aerosol optical depth. Also, the need to expand the memory of the MFRSR data logger is under consideration to extend buffering capabilities when communication to data collectors is not available.

Engineering Change Order ECO-00692, Purchase Data Loggers and Heater Controllers for MFRSRs, is approved and assigned to Gary Hodges. The task is to build and/or procure the spares necessary to support all ACRF MFRSRs/MFRs. Components are on order.

\section{Rotating Shadowband Spectrometer (RSS)}

Mentor: Peter Kiedron, NOAA/ESRL/GMD

Peter Kiedron demonstrated that the rotating shadowband spectrometer (RSS), built by Yankee Environmental Systems, Inc., is capable of providing valuable measurements of direct, diffuse, and global spectral irradiance. Kiedron has also identified problems with the RSS that affect the stability of its calibration and the linearity of its response. Peter has recommended that the RSS be removed from service and sent to him for a complete overhaul. 
Engineering Change Request ECR-00661, RSS Overhaul: Perform Maintenance and Overhaul of RSS, was submitted and approved to perform the upgrade and overhaul of the RSS. Peter Kiedron is the lead. The ARM Working Groups and ARM Science Team Executive Committee (now known as the SISC) recommended the re-engineering of the RSS for implementation in FY2008. A BCR is in process (BCR01457, Defield RSS and Ship to NOAA and Boulder) that has removed and shipped the RSS to NOAA for refurbishment. A data quality report was filed to document the outage and a message is posted on the RSS instrument web page. The disassembly, analysis, reassembly, and characterization of the RSS have identified a series of component and configuration re-engineering issues that were not anticipated. Additional effort and testing are required; parts are on order. The vacuum chamber that houses the CCD was successfully tested. Additional components are being tested prior to final assembly, alignment, and characterization.

\section{Shortwave Spectrometer (SWS)}

Mentor: Connor Flynn, Pacific Northwest National Laboratory

Engineering Change Order, ECO-00697, SWS Baffle, has been approved and assigned to Connor Flynn. This task will redesign and evaluate the optical/measurement of the SWS. The SWS exhibits sensitivity to direct-beam contamination. A new design for the baffle is complete and has been reviewed. Manufacturing and testing of the baffle is approved. This task is being re-scoped.

\subsection{Precipitation and Rain}

\section{Disdrometer (DISDROMETER)}

Mentor: Mary Jane Bartholomew, Brookhaven National Laboratory

There are no open engineering tasks related to the disdrometer instrument suite.

\section{Optical Rain Gauge SGP (ORG)}

Mentor: Mary Jane Bartholomew, Brookhaven National Laboratory

There are no open engineering tasks related to the Optical Rain Gauge (ORG) SGP instrument suite.

Note: There are also ORGs installed on the TWP and AMF metrological towers. Mike Ritsche is the mentor for these systems.

\section{Total Precipitation Sensor NSA (TPS)}

Mentor: Mark Ivey, Sandia National Laboratory

There are no open engineering tasks related to the Total Precipitation Sensor (TPS) instrument suite.

\subsection{Sky Imaging}

\section{Total Sky Imager (TSI)}

Mentor: Vic Morris, Pacific Northwest National Laboratory 
Engineering Change Order ECO-00674, Tasks Associated with TSI Camera and Software Upgrade, is approved for implementation to provide an upgraded camera for the Total Sky Imager (TSI). This ECO is approved with a hold pending the design review to ensure cost and overall impacts are reasonable. An environmental housing for the Axis 211 camera was purchased for testing and design purposes.

Assembly and testing continues.

Engineering Change Order ECO-00644, Subcontract to Upgrade TSI Software, was approved to upgrade the TSI software to allow use of new versions of the Axis camera. Concepts to incorporate the packaging and mechanical design of the new version of the Axis camera will be covered in a new ECR. Progress continues: five of the six tasks are now completed. The final task to refine clear-sky reference images and seasonal azimuth angles is ongoing.

Engineering Change Order ECO-00625, Upgrade TSI Control Boards, was approved and is in process to update the control boards of the TSI- 880 systems. This update will reconcile issues with the real-time clock and power supplies. The subcontract has been placed with Remote Measurement \& Research Company (RMRCo), and the repairs and testing are successfully complete - two boards were repaired and eight additional boards are being fabricated for spares.

\subsection{Surface Fluxes}

\section{Eddy Correlation Station (ECOR)}

Mentor: David Cook, Argonne National Laboratory

Engineering Change Order ECO-00633, Improve Eddy Correlation Station Software, is in process. Tim Martin, in association with David Cook, has proposed to systematically evaluate, document, and reorganize the instrument software to allow for code maintenance and more flexible incorporation of additional logic and sensors, such as the proposed wetness sensor. In addition, the user interface needs to be improved to give access to more debugging and diagnostic messages from the ECOR program. The Version 2.1 software is being installed on the SGP E14 ECOR to document the current configuration of unused analog channels.

Engineering Change Request ECO-00536, Add Wetness Sensors to ECOR System, is in process. Periods of dew, frost, and precipitation often cause data from the $\mathrm{CO}_{2} / \mathrm{H}_{2} \mathrm{O}$ sensor and sonic anemometer to be incorrect. Adding a wetness indication would provide the data user with a more reliable source of information concerning this condition. Wetness sensor testing began at Argonne in mid-January on an ECOR system similar to the ACRF ECORs. Testing so far indicates that different phases of water and types of dew/frost/precipitation produce different voltage levels from the wetness sensor

\section{Energy Balance Bowen Ratio (EBBR) Station}

Mentor: David Cook, Argonne National Laboratory

There are no open engineering tasks related to the EBBR instrument suite.

\section{Soil Water and Temperature System (SWATS)}

Mentor: Daniel Hartsock, University of Oklahoma 
The soil water and temperature system (SWATS), deployed at the SGP site, is designed to provide information about the temperature of the soil and the status of water in the soil profile. Because the SWATS array is aging, the sensor arrays are undergoing a replacement program.

Engineering Change Order ECO-00493, Replace Failing SWATS Sensors, is in process to add new redundant sensor arrays that will be installed at all SGP extended facility sites. These will be installed in a phased manner: five sites per year over 4 years, beginning in 2005 with the sites having multiple failed sensors given highest priority. After the soil recovers from the installation process in 6-12 months, the new sensor array will be connected to the existing SWATS data acquisition system in place of the old sensor array. Sensor arrays for FY2009 from Campbell Scientific have arrived at the SGP and are calibrated and ready for installation. Daniel Hartsock has prepared a status report on the SWATS refurbishment, which is attached to ECO-00493. Baseline Change Request BCR-01508 to update calibration coefficients is complete. The new sensors at E19 were hooked up on December 11, 2008 , and the ingest has been turned back on at both sites. Data now look good for all three SWATS refurbishments. Plans are underway to bury and install new profiles of sensors this spring, starting with E16 (Vici, Oklahoma) in March.

\section{Future Instrumentation Planning}

Instrument planning activities of the ARM Climate Research Facility is a dynamic process driven by the unmet measurement needs of contributing scientists, maintenance, calibration, simulation fidelity, and performance improvement/evolution of instrument and measurement systems. The spatial and temporal configurations of ACRF's instrument systems and associated research sites are reviewed against science/measurement needs - these are important discussions and provide input to future instrument planning. Instrument plan recommendations and priorities are deliberated by the ARM science working groups and vetted thorough the ACRF Infrastructure Management Board for action.

Please contact Jimmy Voyles for additional information related to ACRF instrument planning.

\section{Small Business Innovation Research}

The U.S. Department of Energy (DOE) Small Business Innovative Research (SBIR) web page is available at http://www.er.doe.gov/sbir/. The DOE SBIR program develops instrumentation that can be applied to the science and measurement objectives of the ACRF. 